\title{
Structural investigation of CoMnGe combinatorial epitaxial thin films using microfocused synchrotron X-ray
}

\author{
Yong S. Chu ${ }^{\mathrm{a}, *}$, Andrei Tkachuk ${ }^{\mathrm{a}}$, Stefan Vogt ${ }^{\mathrm{a}}$, Petr Ilinski ${ }^{\mathrm{a}}$, Donald A. Walko ${ }^{\mathrm{a}}$, \\ Derrick C. Mancini ${ }^{\mathrm{a}}$, Eric M. Dufresne ${ }^{\mathrm{b}}$, Liang $\mathrm{He}^{\mathrm{c}}$, Frank Tsui ${ }^{\mathrm{c}}$ \\ ${ }^{a}$ Advanced Photon Source, Argonne National Laboratory, 9700 S. Cass Ave., Argonne, IL 60439-4856, USA \\ ${ }^{\mathrm{b}}$ Department of Physics, University of Michigan, Ann Arbor, MI 48109, USA \\ ${ }^{\mathrm{c}}$ Department Physics and Astronomy, University of North Carolina, Chapel Hill, NC 27599, USA
}

\begin{abstract}
We discuss important experimental considerations and high-throughput synchrotron-based techniques for structural characterization of binary and ternary composition-spread thin films. We apply these techniques to obtain detailed structural phase diagrams of CoMnGe ternary alloy system.
\end{abstract}

(C) 2003 Elsevier B.V. All rights reserved.

PACS: 61.10.Nz; 07.85.Qe; 68.55.Jk

Keywords: CoMnGe; Composition-spread; Phase diagram; X-ray diffraction

\section{Introduction}

In recent years, combinatorial approach has been increasingly adopted to a wide range of disciplines as a method for high-throughput materials discovery [1-3]. In order for combinatorial research to be effective, it is imperative that combinatorial synthesis be accompanied by rapid yet accurate characterization and screening of the physical properties. One of the most essential measurements is structural characterization, because crystalline order-disorder, defects, crystalline phases, texture or strain can significantly affect the functionality of the materials. Furthermore, such information is important for optimizing the synthesis process. However, the limited flux and/or spatial reso-

\footnotetext{
* Corresponding author. Tel.: +1-630-252-0150; fax: +1-630-252-0161.

E-mail address: ychu@aps.anl.gov (Y.S. Chu).
}

lution of laboratory X-ray sources make it difficult to carry out high-throughput structural characterization on combinatorial samples. In particular, the structural phase mapping of composition-spread thin films is especially challenging because the measurement requires both high flux density and spatial resolution. In order to eliminate this bottleneck in combinatorial research, we have developed synchrotron-based instrumentation and techniques for structural characterization. In this paper, we illustrate the important experimental factors relevant for characterizing the two most common types of combinatorial samples, binary and ternary composition-spreads, and report detailed structural phase diagrams of CoMnGe ternary alloy system grown epitaxially on Ge $\left(\begin{array}{lll}1 & 1 & 1\end{array}\right)$ substrate using combinatorial MBE techniques [2,3]. This ternary alloy system is of great technological interest, because it exhibits robust magnetic properties [2,3], including potential half-metals, e.g. $\mathrm{Co}_{2} \mathrm{MnGe}$ [4], 
and the synthesis of many alloys in the system can be compatible with semiconductor processing. This combination makes the system an excellent choice for exploring spintronics materials and applications.

\section{Experimental}

\subsection{Samples}

Two composition-spread samples were studied, a binary sample of $\left(\mathrm{Co}_{x} \mathrm{Mn}_{1-x}\right)_{0.75} \mathrm{Ge}_{0.25}$ grown on Ge (1 111 ) substrate, and a ternary sample of $\mathrm{Co}_{x} \mathrm{Mn}_{y} \mathrm{Ge}_{1-x-y}$ grown also on Ge (lllll). Synthesis was carried out using an advanced combinatorial MBE system [3]. A combination of computer-controlled sample rotation, masking, and source shutters was used to execute the combinatorial synthesis. A linear gradient thickness profile (wedge) for each of the precursors, i.e. Co, $\mathrm{Mn}$ and $\mathrm{Ge}$, was produced during deposition by moving a precision shadow mask across the substrate. The 'height' of each wedge was about a monolayer. The precursors were deposited sequentially to form a trilayer, and the trilayers were repeated to produce a thick film. Co and Ge were evaporated from e-beam hearths, and Mn was from an effusion cell. The two samples were grown at $250{ }^{\circ} \mathrm{C}$ and annealed at $450{ }^{\circ} \mathrm{C}$. Epitaxial processes were monitored in real time by scanning reflection high-energy electron diffraction (RHEED). The binary sample is about $1 \mathrm{~cm}$ long and $0.5 \mathrm{~cm}$ wide, and the ternary sample is diamond shaped and about $1 \mathrm{~cm}^{2}$. The nominal film thickness for the binary sample is $500 \AA$, and that for the ternary sample is $600 \AA$.

\subsection{Structural characterization}

In order to map out phase diagrams using composition-spread combinatorial samples, careful attention must be given to the spatial resolution of the X-ray probe. Since binary or ternary samples are typically about $1 \mathrm{~cm}$ in dimension, a composition resolution of about $1 \%$ requires a beam's footprint (illuminated area) on the sample to be about $100 \mu \mathrm{m}$ or less in dimension. This argument is, of course, based on the assumption that the composition gradient is uniform and no abrupt phase separation exists in the sample. Furthermore, high quality single-crystalline films would require additional considerations for measuring three-dimensional (3D) structures, because these measurements would involve rotating the sample with respect to the incident beam that would change the size and shape of the footprint. Fig. 1 illustrates how sensitively the beam's footprint depends on the magnitude and direction of the momentum transfer vector $\boldsymbol{q} \equiv \boldsymbol{k}_{\mathrm{f}}-\boldsymbol{k}_{\mathrm{i}}$, and thus the reciprocal lattice vector $\boldsymbol{g}=(h, k, l)$, where $\boldsymbol{k}_{\mathrm{i}}$ and $\boldsymbol{k}_{\mathrm{f}}$ are the incident and diffracted wavevectors, respectively [5]. A brief discussion about the beam's footprints of three pertinent cases is given below (Fig. 1(b)-(d)).

In case 1 (Fig. 1(b), the family of reflections $(h, h, h)$, such as $\left(\begin{array}{lll}1 & 1 & 1\end{array}\right)$, has only a component perpendicular to the surface (i.e. $\boldsymbol{q}=\boldsymbol{q}_{\text {perp }}$ ), and the diffraction conditions can be satisfied regardless of the azimuthal orientation of the sample with respect to the surface-normal. In contrast the footprint for the (lill 111$)$ reflection (case 2, Fig. 1(c)) is significantly broadened compared to that for (1 111 ) owing to the smaller incident angle of the beam with respect to the sample

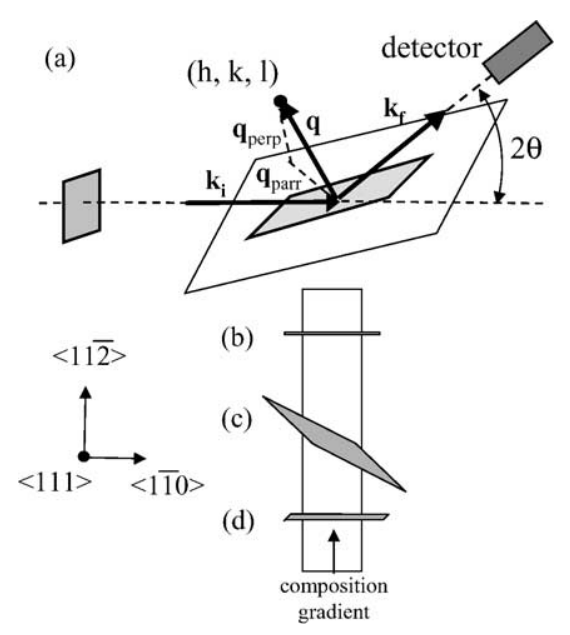

Fig. 1. Schematic diagrams of: (a) X-ray diffraction geometry and (b)-(d) the footprint of the beam on the sample. In (a) $\boldsymbol{k}_{\mathrm{i}}$ and $\boldsymbol{k}_{\mathrm{f}}$ are the incident and the diffracted wavevectors, respectively. The momentum transfer wavevector is defined as $\boldsymbol{q} \equiv \boldsymbol{k}_{\mathrm{f}}-\boldsymbol{k}_{\mathrm{i}}$. $2 \theta$ is the angle of the diffracted beam. $\boldsymbol{q}_{\text {perp }}$ and $\boldsymbol{q}_{\text {parr }}$ are the respective perpendicular and parallel components of $\boldsymbol{q}$ with respect to the sample surface. The footprint of the beam on the sample surface is indicated by a shaded parallelogram. Sections (b)-(d) are the simulated footprints of a $0.3 \mathrm{~mm}$ (horizontal) by $1 \mathrm{~mm}$ (vertical) incident beam for (llll 111$),\left(\begin{array}{lll}1 & 1 & 1\end{array}\right)$, and (llll 113$)$ diffraction conditions of a FCC binary sample whose crystallographic directions and the composition-gradient are indicated by the arrows. 
surface. In addition, it also runs diagonally with respect to the composition gradient owing to the azimuthal orientation required by the diffraction condition. By carefully considering these factors, the broadening of the footprint can be controlled by choosing certain set of reflections. Case 3 is one such example as shown in Fig. 1(d). By choosing the $\left(\begin{array}{lll}1 & 1 & 3\end{array}\right)$ reflection, the footprint is predominantly broadened along the $\langle\overline{1} 10\rangle$ direction perpendicular to the binary composition gradient (along the $\langle 11 \overline{2}\rangle$ ), thus gives rise to a good composition resolution.

Diffraction measurements on the binary sample $\left(\mathrm{Co}_{x} \mathrm{Mn}_{1-x}\right)_{0.75} \mathrm{Ge}_{0.25}$, was carried out at the bending magnet beamline 2-BM-B of the Advanced Photon Source (APS) at Argonne National Laboratory using a standard four-circle diffractometer. We manipulated the beam's footprint based on the above discussion in order to obtain a few percent composition resolution from an unfocused incident beam narrowed down with silts to about $0.3 \mathrm{~mm} \times 2 \mathrm{~mm}$. However, manipulating such a large footprint would not be useful for characterizing a ternary sample. Furthermore, highthroughput measurements on a ternary sample would require $2 \mathrm{D}$ microfocusing rather than a pinhole in order to maintain sufficient flux density. Therefore, for investigating the ternary sample, $\mathrm{Co}_{x} \mathrm{Mn}_{y} \mathrm{Ge}_{1-x-y}$, we employed Kirkpatrick-Baez (KB) mirrors [6] to produce a microfocused X-ray beam of $5 \mu \mathrm{m}$ (horizontal FWHM) $\times 12 \mu \mathrm{m}$ (vertical FWHM) at an undulator beamline, 7-ID-C, of the APS. The size of the beam was kept deliberately much larger than the minimal spot size for this particular instrument $(\sim 1 \mu \mathrm{m})$ in order to retain reasonably high angular resolution for the diffraction measurements and a reasonably long focal length $(\geq 50 \mathrm{~cm})$ to leave enough space for the wide range of sample rotation necessary for reciprocal mapping. During the experiment, an inert $\mathrm{N}_{2}$ environment around the sample was maintained using a thin plastic bag, in order to avoid possible sample oxidation caused by the intense X-ray beam.

During the diffraction experiments, we also carried out X-ray fluorescence measurements in parallel using the same sample coordinate system, in order to determine the composition simultaneously. The use of a compact Peltier-cooled Si drift diode energy-dispersive detector [7] for fluorescence measurement rather than a commonly used liquid nitrogen-cooled $\mathrm{Ge}$ detector provided increased experimental flexibility needed to carry out both measurement in parallel. The parallel measurements of the composition and structure under the same experimental setup is crucial not only for achieving high-throughput characterization but also for eliminating any systematic experimental errors associated with transporting samples between two separate experimental stations.

\section{Results and discussion}

\subsection{Diffraction patterns of CoMnGe grown on $\mathrm{Ge}\left(\begin{array}{lll}1 & 1 & 1\end{array}\right)$}

The CoMnGe alloy films grown on Ge $\left(\begin{array}{lll}1 & 1 & 1\end{array}\right)$ exhibit two crystalline phases, a hexagonal phase and a face-centered cubic (FCC) phase, both of which are epitaxial to the Ge substrate and anisotropically strained due to epitaxy. A schematic diagram of the observed diffraction patterns are shown in Fig. 2.

In order to conveniently express the epitaxial relationship between the alloy phases and the substrate and appropriately account for their anisotropic strain, we employ a hexagonal surface coordinate system [8], whose $a$ - and $b$-axes are parallel to, and $c$-axis is perpendicular to the substrate surface. In this hexagonal coordinate system, the extended unit cell for the Ge substrate has a hexagonal structure with the lattice parameters, $a=a_{0} / \sqrt{ } 2$ and $c=\sqrt{ } 3 a_{0}$, where $a_{0}$ is the cubic lattice constant of Ge. Consequently, the reciprocal space of the Ge substrate can be indexed using hexagonal indices, $(H, K, L)_{h}$, which are related to the conventional cubic indices, $(h, k, l)$, through the following coordinate transformation matrix.

$$
\begin{aligned}
\left(\begin{array}{l}
H \\
K \\
L
\end{array}\right) & =\left(\begin{array}{ccc}
-1 / 2 & 1 / 2 & 0 \\
0 & -1 / 2 & 1 / 2 \\
1 & 1 & 1
\end{array}\right)\left(\begin{array}{l}
h \\
k \\
l
\end{array}\right), \\
\text { or }\left(\begin{array}{l}
h \\
k \\
l
\end{array}\right) & =\left(\begin{array}{ccc}
-4 / 3 & -2 / 3 & 1 / 3 \\
2 / 3 & -2 / 3 & 1 / 3 \\
2 / 3 & 4 / 3 & 1 / 3
\end{array}\right)\left(\begin{array}{l}
H \\
K \\
L
\end{array}\right)
\end{aligned}
$$

For example, $\left(\begin{array}{lll}1 & 1 & 1\end{array}\right)_{c}=\left(\begin{array}{lll}0 & 0 & 3\end{array}\right)_{h},\left(\begin{array}{lll}1 & 1 & 1\end{array}\right)_{c}=\left(\begin{array}{llll}1 & 0 & 1\end{array}\right)_{h}$, $\left(\begin{array}{llll}0 & 2 & 2\end{array}\right)_{c}=\left(\begin{array}{llll}1 & 0 & 4\end{array}\right)_{h},\left(\begin{array}{llll}0 & 0 & 2\end{array}\right)_{c}=\left(\begin{array}{lll}0 & 1 & 2\end{array}\right)_{h}$, and $\left(\begin{array}{llll}1 & 1 & 3\end{array}\right)_{c}=$

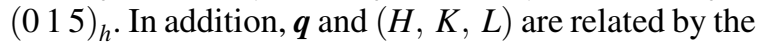
relationship, $\left|\boldsymbol{q}_{\text {perp }}\right|=(2 \pi / c) L$ and $\left|\boldsymbol{q}_{\text {parr }}\right|=(2 \pi / a)$ $\sqrt{4\left(H^{2}+H K+K^{2}\right) / 3}$. For convenience, the rest 


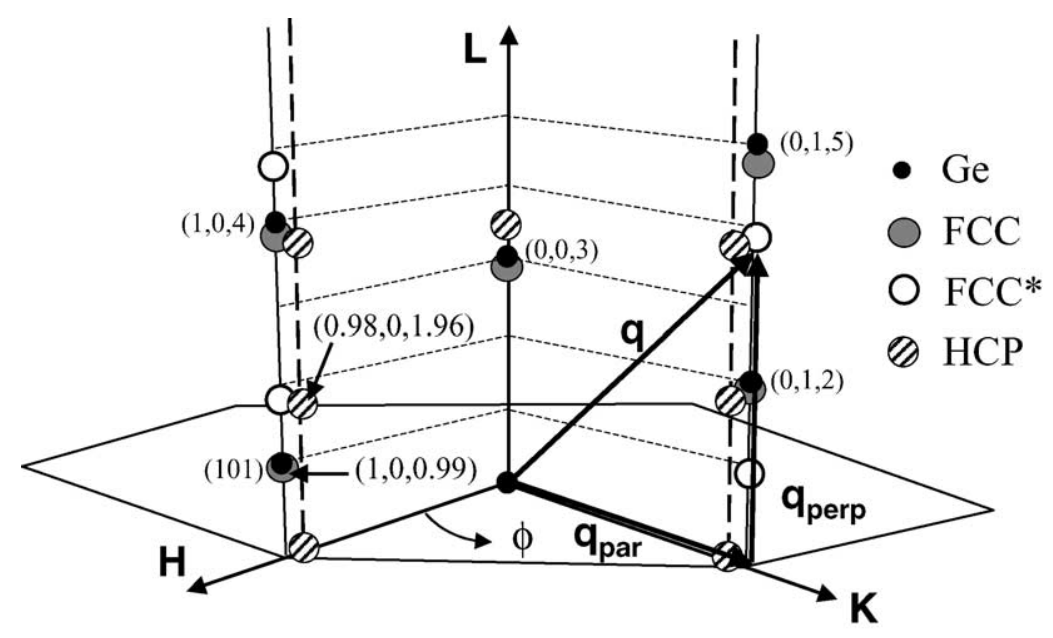

Fig. 2. Schematic diffraction patterns of CoMnGe alloys grown on Ge $\left(\begin{array}{lll}1 & 1 & 1\end{array}\right)$ represented in a hexagonal coordinate system discussed in the text. Circles represent diffraction spots for various phases: shaded large circles for single-crystalline FCC; empty large circles for its rotated twin domain; hatched circles for hexagonal phase; and small circles for the Ge substrate.

of this paper uses only the hexagonal coordinate system of the substrate without the subscript.

We now return to the schematic diffraction patterns shown in Fig. 2. For reference, we first focus on the diffraction pattern of the Ge substrate, which is represented by the small solid circles. As Ge crystal exhibits the FCC symmetry with an $\mathrm{ABC}$ stacking sequence along the $L$-direction (i.e. the $\left\langle\begin{array}{llll}1 & 1 & 1\end{array}\right\rangle$ direction in the cubic system) [5], all reflections along the $L$-direction are separated by $\Delta L=3$, thus exhibiting a three-fold rotation symmetry about the $L$-axis.

The FCC reflections (the shaded circles) nearly coincide those of the Ge. The difference between diamond structure of Ge and FCC structure of the film only shows up at higher order reflections and is not shown in Fig. 2. Since we use a coordinate system "scaled" for the substrate lattice, the position of the film reflection actually measures the ratio between the parameters of the substrate and film. For example, the FCC reflection at $(1,0,0.98)$ indicates that $a_{\mathrm{Ge}} / a_{\mathrm{FCC}}=1$ and $c_{\mathrm{Ge}} / c_{\mathrm{FCC}}=0.98$, i.e. pseudomorphic in the growth plane and tensile strain along the $L$-axis.

In reality, however, an additional FCC pattern (the empty circles) exists due to the existence of a twin domain [9], which is rotated by $60^{\circ}$ about the $L$-axis but otherwise identical to the other FCC structure. Consequently, the diffraction pattern is also rotated by $60^{\circ}$ about the $L$-axis, and thus making the two FCC reflections at $(1,0,0.99)$ and $(0,1,0.99)$ equivalent. Understanding the diffraction pattern of the rotated twin domain is crucial to making accurate measurement of the epitaxial thin film whose structure is very close or equal to the substrate structure, because the measurement of reflections belonging to the rotated domain can be made without being overwhelmed by substrate reflections that are several orders of magnitude more intense.

The diffraction pattern of the hexagonal phase (the hatched circles in Fig. 2) reflects an $\mathrm{AB}$ stacking sequence leading to reflections at 'even' increments along the $L$-axis [5]. For example, along a line parallel to $L$ and through $H=0.97$ and $K=0$, reflections occur at an increment of $\Delta L=1.96$, while along the $L$ axis, the reflections occur at an increment of $2 \Delta L$. Again, the position of the reflection at $H=0.97$ and $L=1.96$ indicates that the respective ratio of the $\mathrm{Ge}$ unit cell to that of the hexagonal phase along the directions parallel and perpendicular to the surface is 0.97 and 1.96. Using this difference in the symmetry of the diffraction patterns, we can unequivocally distinguish the two different crystalline phases regardless of the magnitude of the anisotropic epitaxial strain.

It is important to point out that the CoMnGe orthorhombic phase $(a=5.894, b=3.798$, and 
$c=7.035 \AA$ ) [10], which is a stable bulk alloy, was not observed in this thin-film system. We attribute this to the low symmetry of the orthorhombic phase that cannot be stabilized on this Ge surface.

\subsection{Structural phase diagram of the binary sample}

Structural phase diagram of the binary sample $\left(\mathrm{Co}_{x} \mathrm{Mn}_{1-x}\right)_{0.75} \mathrm{Ge}_{0.25}$ has been examined by making a series of scans along $L$ ( $L$-scans) from 3.4 to 4.3 at $H=0$ and $K=1$, and the results are shown in Fig. 3. The scans were chosen because both hexagonal and FCC phases can be detected without nearby substrate background, and the beam footprint on the sample was small. Since the phase diagram shown in Fig. 3 was obtained by $L$-scans, it measures structural component normal to the surface, while being insensitive to the small structural changes parallel to the surface. The Co concentration $x$ shown on the top of the plot is estimated from the growth parameters and confirmed later with the composition measurement carried out on the ternary sample. The regions of the sample below $x=0$ and above $x=100 \%$ correspond to $\mathrm{Mn}_{0.75} \mathrm{Ge}_{0.25}$ and $\mathrm{Co}_{0.75} \mathrm{Ge}_{0.25}$ binary alloys, respectively.

The diffraction phase map (Fig. 3) reveals four local intensity maxima, centered near $L=3.82,3.55,3.7$, and 4.0. The phase with the highest diffraction intensity around $L=4$ is FCC, while the others are hexagonal. For convenience, we refer to these three hexagonal structures as $\mathrm{H} 1, \mathrm{H} 2$ and $\mathrm{H} 3$, in order of increasing Co concentration. The FCC and HCP phases shown here correspond to $\left(\begin{array}{lll}0 & 2 & 2\end{array}\right)$ and $\left(\begin{array}{lll}1 & 0 & 2\end{array}\right)$ reflections when indexed according to their native crystal coordinates.

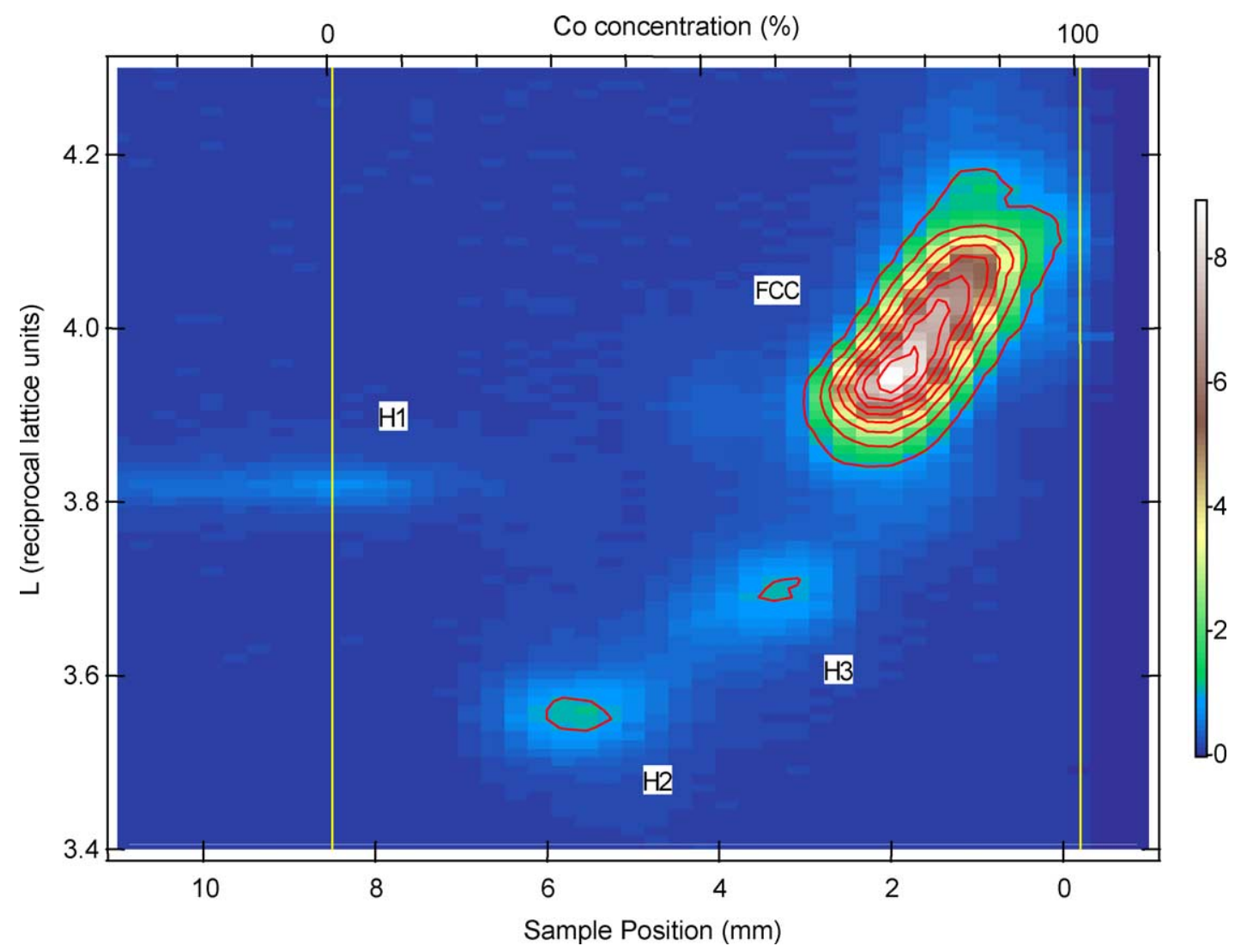

Fig. 3. Structural phase diagram of $\left(\mathrm{Co}_{x} \mathrm{Mn}_{1-x}\right)_{0.75} \mathrm{Ge}_{0.25}$ binary sample. $\mathrm{Mn}_{0.75} \mathrm{Ge}_{0.25}$ and $\mathrm{Co}_{0.75} \mathrm{Ge}_{0.25}$ phases are below $x=0$ and above $x=100 \%$, respectively. 
Up to about $x=20 \%$, the $L$-position of the H1 phase remains almost constant. This observation indicates that the $\mathrm{H} 1$ structure is stable under the $\mathrm{Co}-\mathrm{Mn}$ substitution in the initial Mn-Ge alloy, suggesting up to about $15 \%$ Co solubility into this hexagonal phase. Among the three hexagonal phases, the $\mathrm{H} 1$ phase has the least degree of in-plane lattice matching with the substrate, about $4 \%$ larger than the substrate lattice. The phase transition from $\mathrm{H} 1$ to $\mathrm{H} 2$ near $x=20 \%$ is abrupt, with a sudden increase of the out-of-plane lattice parameter (i.e. decrease in $L$-position). Like $\mathrm{H} 1$, the lattice of $\mathrm{H} 2$ normal to the surface remains almost constant up to about $x=40 \%$. Among the three hexagonal phases, $\mathrm{H} 2$ exhibits the best in-plane lattice matching with the substrate, about $1 \%$ larger than that of the substrate. Contrary to the two other hexagonal phases, the out-of-plane lattice parameter of $\mathrm{H} 3$ decreases almost linearly up to the hexagonalto-FCC phase transition near $x=60 \%$. At $x=55 \%$, the in-plane and out-of-plane lattice parameters, $a$ and $c$, are nearly identical to those of the hexagonal CoGeMn bulk alloy [10].

Some portion of the FCC phase coexists with the H3 phase due to a rather sluggish phase-transition. The FCC phase is structurally quite different from the rest of the phases. Its surface-normal lattice parameter is linear with respect to Co composition, while its inplane lattice is nearly matched with that of the substrate to better than 1\%. In fact for $x$ from 80 to $95 \%$, its in-plane lattice is matched with that of the substrate indicating the absence of strain relaxation. Moreover, the film is completely lattice matched with the substrate in both directions at $x=83 \%$.

The transition from $\mathrm{H} 3$ to a high quality epitaxial FCC structure correlates with a magnetic transition to a region of robust ferromagnetism with high moment, high Curie temperature, and large magneto-optic effects [3]. Such correlation is not unexpected since magnetic properties correlate strongly with the symmetry of electronic and crystalline structures $[11,12]$. In particular, $x=67 \%$ corresponds to the stoichiometry of the predicted half-metal $\mathrm{Co}_{2} \mathrm{MnGe}$ [4].

\subsection{Structural phase diagram of the ternary sample}

Structural phase diagrams of the ternary sample, $\mathrm{Co}_{x} \mathrm{Mn}_{1-y} \mathrm{Ge}_{1-x-y}$ have been studied by performing a series of $L$-scans as a function of 2D sample position with $0.4 \mathrm{~mm}$ increments. X-ray fluorescence measurements were carried out before and during the diffraction experiments, in order to establish independently the coordinate transformation matrix from sample position to ternary composition. Quantitative analysis of the X-ray fluorescence indicates that the composition gradients are linear with no detectable macroscopic phase separation. Details of the X-ray fluorescence analysis are given elsewhere [13]. Three slices at three fixed Ge concentrations of 27,45 , and $64 \%$ from this multi-dimensional data set containing ternary composition and diffraction intensity are presented here, as shown in Fig. 4. These results are intended to show effects of Ge concentration on the ternary system.

As shown in Fig. 4(a), the structural phase diagram at $27 \%$ Ge [owing to the lack of sampling resolution $(0.4 \mathrm{~mm})$ this is presented instead of that at $25 \% \mathrm{Ge}$ ] resembles that of the binary sample with $25 \% \mathrm{Ge}$. It reproduces all the major phases and transitions observed in the binary sample. In particular, excellent agreement is found in $\mathrm{H} 1$ and FCC between the two experiments. However, some differences also exist between the two, such as the intensity of the H2, H3, and FCC phases. The structural phase diagram obtained from the ternary sample using the microbeam exhibits intricate intensity features for the $\mathrm{H} 3$ and FCC phases, suggesting more complex structural dependence on the local alloy composition, which needs to be further investigated with a higher sampling intervals than $0.4 \mathrm{~mm}$ used in this experiment. These effects may also explain the enhanced intensity observed in $\mathrm{H} 2$ and $\mathrm{H} 3$ from the ternary sample compared to that of the FCC phase.

At $45 \% \mathrm{Ge}$, the diffraction intensity of the FCC phase increases almost five times due to increased crystalline ordering of this phase (Fig. 4(b)). The inplane scan through this phase indicates a considerable decrease in the peak width. On the other hand, H1 loses intensity significantly indicating a reduction in crystalline ordering. One salient feature of this structural phase diagram is that the out-of-plane lattice parameter of all the phases, except HCP1, decreases linearly with increasing Co concentration.

This dramatic linear response for the Mn-Co substitution is almost absent at $64 \%$ Ge (Fig. 4(c)). Moreover, all phases become much weaker in intensity, 

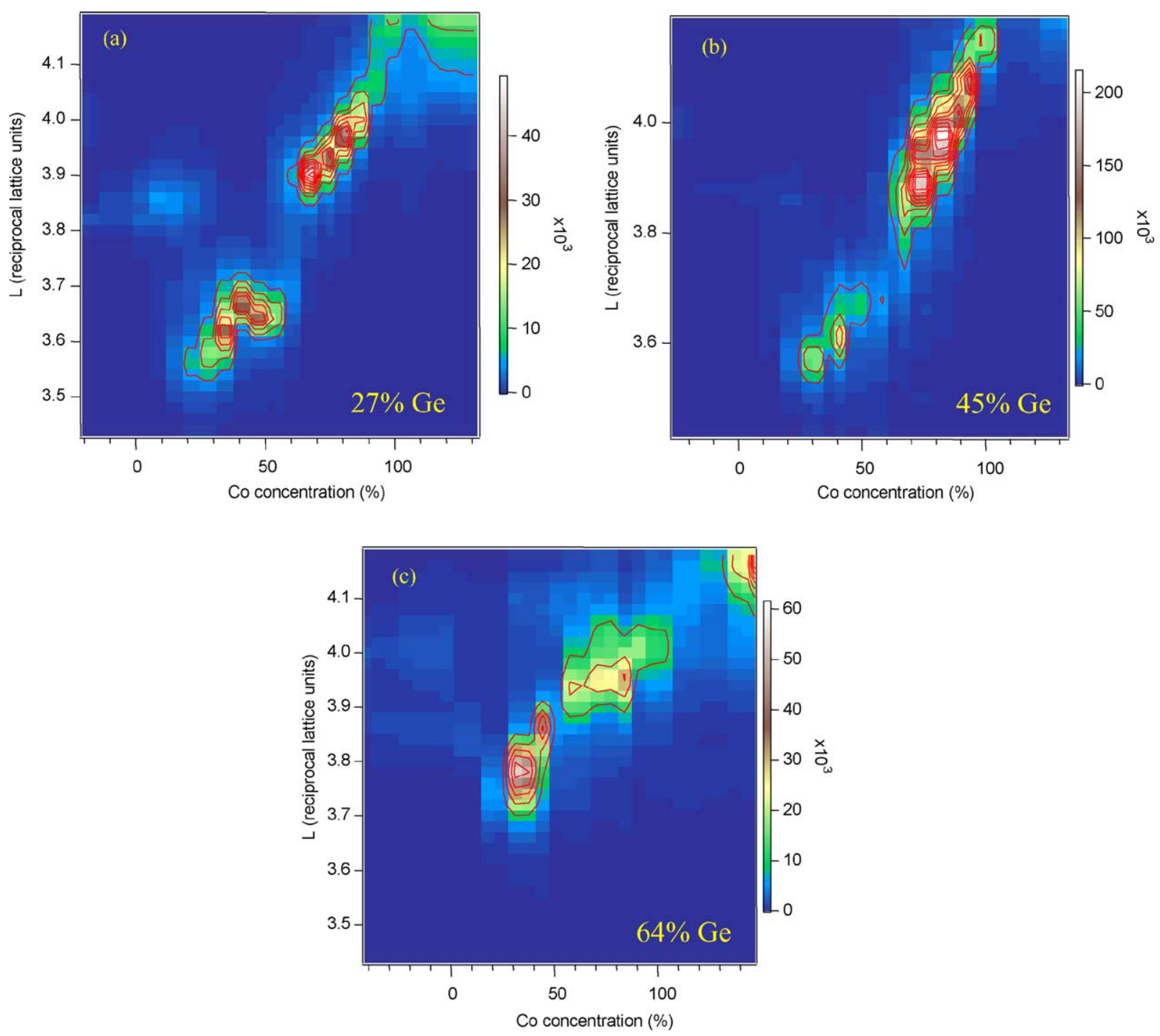

Fig. 4. The binary phase diagrams of: (a) $\left(\mathrm{Co}_{x} \mathrm{Mn}_{1-x}\right)_{0.73} \mathrm{Ge}_{0.27}$, (b) $\left(\mathrm{Co}_{x} \mathrm{Mn}_{1-x}\right)_{0.55} \mathrm{Ge}_{0.45}$ and (c) $\left(\mathrm{Co}_{x} \mathrm{Mn}_{1-x}\right)_{0.36} \mathrm{Ge}_{0.64}$ obtained from the ternary sample, $\mathrm{Co}_{x} \mathrm{Mn}_{1-y} \mathrm{Ge}_{1-x-y}$ grown on $\mathrm{Ge}\left(\begin{array}{lll}1 & 1 & 1\end{array}\right)$.

indicating a suppression of crystalline ordering. In addition, the separation between $\mathrm{H} 2$ and $\mathrm{H} 3$ become very blurred. The intensity for $\mathrm{H} 1$ phase nearly disappears, while a new FCC structure emerges in the MnGe binary alloys.

Based on these limited structural phase diagrams, we can make several observations about the CoMnGe on Ge (lllll 111 epitaxial system. First, the three hexagonal phases appear to exhibit the highest crystalline order at low Ge concentration, while the FCC phase appears to be much more ordered near $50 \%$ Ge. Further increase of $\mathrm{Ge}$ concentration decreases crystalline ordering for all epitaxial phases. Second, the stability of a phase is controlled by the epitaxial constraints imposed by the substrate. Specifically, the triangular arrangement of the Ge $\left(\begin{array}{lll}1 & 1 & 1\end{array}\right)$ surface favors structures with three- or six-fold symmetries, i.e. hexagonal and FCC phases. The absence of an orthorhombic phase supports this finding. Our preliminary investigation on the CoMnGe on Ge (lllll) system 
reveals quite different structural phase diagrams, again, also due to the symmetry of the substrate.

\section{Summary}

We have described important experimental factors in carrying out structural characterization of combinatorial samples using either an unfocused or microfocused synchrotron X-ray beam. Effects of X-ray beam's footprint and the technique for simultaneous measurements of both composition and crystalline structure have been discussed. The techniques have been applied to study binary and ternary compositionspreads of CoMnGe grown epitaxially on Ge (llll). This ternary epitaxial system exhibits interesting structural phases and transformations that are stabilized by the structure and symmetry of the substrate. And finally, the use of microfocused X-ray beam has made it possible to map the entire ternary phase diagram from a single sample without sacrificing composition resolution, while providing the necessary flux density.

\section{Acknowledgements}

Use of the Advanced Photon Source was supported by the US Department of Energy, Office of Science Basic Energy Sciences, under Contract No. W-31-109ENG-38. The combinatorial research at University of North Carolina is supported in part by NSF DMR-0108605. We thank Jesse Guzman of University of Michigan for experimental assistance.

\section{References}

[1] E.J. Amis, X.-D. Xiang, J.-C. Zhao, MRS Bull. 27 (4), (April, 2002), pp. 295-297 (and the references therein).

[2] Y.K. Yoo, F. Tsui, MRS Bull. 27 (4) (2002) 316-323.

[3] F. Tsui, L. He, L. Ma, in: I. Takeuchi, et al. (Eds.), Proceedings of the MRS Symposium on Combinatorial and Artificial Intelligence Methods of Materials Science, vol. 700, MRS, Pittsburgh, PA, 2001, pp. S2.2.1-S2.2.6.

[4] S. Ishida, S. Fujii, H. Nagayoshi, S. Asano, Phys. B 254 (1998) 157-165.

[5] B.E. Warren, X-ray Diffraction, Dover Publications, Inc., New York, 1990.

[6] P.J. Eng, M. Newville, M.L. Rivers, S.R. Sutton, SPIE Proc. 3449 (1998) 145.

[7] J.S. Iwanczyk, B.E. Patt, J. Segal, J. Plumnner, G. Vikelis, B. Hedman, K.O. Hodgson, A.D. Cox, L. Rehn, J. Metz, Nucl. Instrum. Methods A 380 (1996) 288-294.

[8] Y.S. Chu, I.K. Robinson, A.A. Gewirth, Phys. Rev. B 55 (1997) 7945-7954.

[9] Y.S. Chu, I.K. Robinson, A.A. Gewirth, J. Chem. Phys. 110 (1999) 5952-5959.

[10] P. Villars, A. Prince, H. Okamoto, Handbook of Ternary Alloy Phase Diagrams, ASM International, Materials Park, OH, 1995.

[11] Z.Q. Liu, S.D. Bader, Rev. Sci. Instrum. 71 (2000) 1243.

[12] Y.K. Yoo, T. Ohnishi, G. Wang, F. Duewer, X.-D. Xiang, Y.S. Chu, D.C. Mancini, Y.-Q. Li, R.C. O'Handley, Intermetallics 9 (2001) 541.

[13] S. Vogt, Y.S. Chu, A. Tkachuk, P. Ilinski, F. Tsui, Appl. Surf. Sci. 223 (2004) 214-219. 